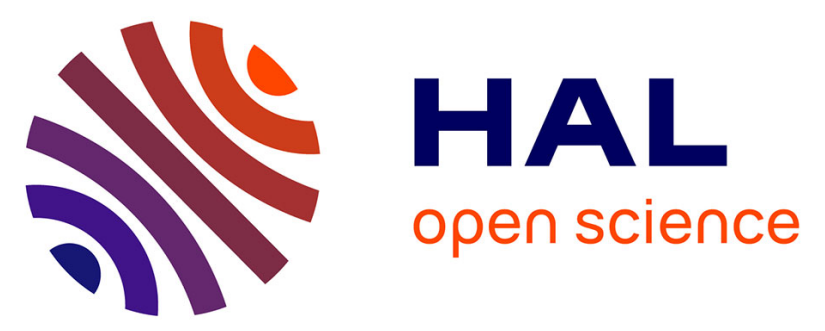

\title{
Évaluation de l'écotoxicité intrinsèque à l'aide d'une batterie de tests d'écotoxicité réalisés après une lixiviation X30-402
}

B. Ferrari, J.F. Férard

\section{- To cite this version:}

B. Ferrari, J.F. Férard. Évaluation de l'écotoxicité intrinsèque à l'aide d'une batterie de tests d'écotoxicité réalisés après une lixiviation X30-402. Environnement, Ingénierie \& Développement, 1999, N¹6 - 4ème trimestre 1999, pp.42-44. 10.4267/dechets-sciences-techniques.1065 . hal03181746

\author{
HAL Id: hal-03181746 \\ https://hal.science/hal-03181746
}

Submitted on 25 Mar 2021

HAL is a multi-disciplinary open access archive for the deposit and dissemination of scientific research documents, whether they are published or not. The documents may come from teaching and research institutions in France or abroad, or from public or private research centers.
L'archive ouverte pluridisciplinaire HAL, est destinée au dépôt et à la diffusion de documents scientifiques de niveau recherche, publiés ou non, émanant des établissements d'enseignement et de recherche français ou étrangers, des laboratoires publics ou privés.

\section{(c)(1)}

Distributed under a Creative Commons Attribution| 4.0 International License 


\title{
ÉVALUATION DE L'ÉCOTOXICITÉ INTRINSÈQUE À L'AIDE D'UNE BATTERIE DE TESTS D'ÉCOTOXICITÉ RÉALISÉS APRĖS UNE LIXIVIATION X30-402
}

\author{
B. Ferrari - J.F. Férard \\ Centre des sciences de l'environnernent
}

Cette étude entre dans le cadre du programme de recherche sur « l'écocompatibilité des déchets » financé par l'Ademe et piloté par Polden. Les expérimentations ont été réalisées sur des mâchefers d'incinération d'ordures ménagères de type $V$ (MIOM) et sur des scories de seconde fusion au Plomb (Sc2Pb).

\section{OBJECTIFS}

Un premier objectif est d'évaluer l'écotoxicité intrinsèque de 2 déchets différents selon une stratégie d'évaluation bien définie. Cette stratégie fait appel à une approche directe et indirecte. L'évaluation de la toxicité par mesure directe consiste en un contact direct des organismes avec la matrice déchet. Par contre, dans les tests de toxicité indirecte, les organismes sont mis en présence d'un lixiviat. Un deuxième objectif est de comparer l'écotoxicité d'un lixiviat de rapport $L / S$ de 2 (R2) et d'un lixiviat de rapport L/S de 10 (RIO).

\section{MÉTHODOLOGIE}

Chaque déchet a été testé directement ou indirectement à l'aide d'une batterie de tests de toxicité présentés dans le tableau I. Les différents tests utilisés ont été choisis parce qu'ils sont sensibles, utilisables avec peu de matrice liquide ou solide et parce qu'ils permettent d'obtenir des réponses à la fois en terme de toxicité aiguë, de toxicité chronique et de génotoxicité.

Les lixiviations ont été réalisées selon la norme Afnor $\times 30-402^{[10]}$ et 2 rapports liquide/solide (R2 et R I0). Au bout de 24 heures, la lixiviation a été arrêtée et les échantillons de MIOM ou de Sc2Pb ont été décantés pendant 15 minutes puis centrifugés à $3500 \mathrm{tr} / \mathrm{min}$ pendant 10 minutes à température ambiante. Le surnageant a alors été récupéré puis directement testée après mesure du $\mathrm{pH}$ et de la conductivité.s

\section{RÉSULTATS}

\section{Cas des MIOM (tableau 2)}

- Le test Algue est le test le plus sensible pour les MIOM quelque soit le rapport L/S choisi, le test Algue est donc un bon indicateur de la toxicité pour ces mâchefers.

- Les résultats sont remarquablement concordants entre les lixiviats R2 et R10. Si l'on considère l'ensemble des 8

\begin{tabular}{|c|c|c|c|c|}
\hline Test & Espèce & Durée d'exposition & Critère & Référence \\
\hline \multicolumn{5}{|l|}{ Contact direct } \\
\hline Plante & Lactuca sativa & 14 jours & $\begin{array}{l}\text { Effets sur la germination, } \\
\text { les poids frais et secs }\end{array}$ & [1] \\
\hline Microtox phase solide & Vibrio fisheri & $20 \mathrm{~min}$. & Inhibition de la luminescence & [2] \\
\hline \multicolumn{5}{|l|}{ Contact indirect } \\
\hline Ames fluctuation & $\begin{array}{l}\text { Salmonella typhirnurium } \\
\text { souche TA98 et TAI } 100\end{array}$ & 5 jours & $\begin{array}{l}\text { Induction des mutations } \\
\text { reverses avec et sans } 59\end{array}$ & [3] \\
\hline Mutatox & Vibrio fisheri (mutant) & 20 et $24 \mathrm{~h}$ & $\begin{array}{l}\text { Induction des mutations } \\
\text { reverses avec et sans } 59\end{array}$ & [4] \\
\hline Microtox & Vibrio fisheri & 15,30 , et $60 \mathrm{~min}$ & Inhibition de la luminescence & [5] \\
\hline Cladocère & Daphnia magna & $24,48 \mathrm{~h}$ & Inhibition du déplacement & [6] \\
\hline \multirow[t]{2}{*}{ Cladocère } & Ceriodaphnia dubia & 7 jours & Effet sur la reproduction & [7] \\
\hline & $\begin{array}{l}\text { Pseudokirchneriella } \\
\text { subcapitata }\end{array}$ & $72 \mathrm{~h}$ & Inhibition de la croissance & [8] et [9] \\
\hline Plante & Lactuca sativa & 14 jours & $\begin{array}{l}\text { Effets sur la germination, } \\
\text { les poids frais et secs }\end{array}$ & [7] \\
\hline
\end{tabular}


Tableau 2 : Résultats physico-chimiques et écotoxicologiques sur les MIOM

\begin{tabular}{|c|c|c|c|c|c|}
\hline \multicolumn{2}{|c|}{ Contact direct } & \multicolumn{2}{|c|}{ Mode de calcul des unités toxiques UT } & \multicolumn{2}{|c|}{ MIOM solide } \\
\hline \multicolumn{2}{|c|}{ Microtox 20 min. $(n=2)$} & \multicolumn{2}{|c|}{ 100/CE50 } & \multicolumn{2}{|c|}{$83(0,6 \%)$} \\
\hline $\begin{array}{l}\text { Test plante }(n=1) \\
14 \text { jours }\end{array}$ & $\begin{array}{l}\text { Germination } \\
\text { Poids frais } \\
\text { Poids sec }\end{array}$ & \multicolumn{2}{|c|}{$\begin{array}{l}1100 / \text { LOEC } \\
\text { 100/LOEC } \\
\text { 100/LOEC }\end{array}$} & \multicolumn{2}{|c|}{$\begin{array}{l}5.6 \\
3,1\end{array}$} \\
\hline Contact indirect & \multicolumn{2}{|c|}{ Mode de calcul des unités toxiques UT } & MIOM R2 & MIOM RIO & R2/R10 \\
\hline $\mathrm{pH}(n=1 \mathrm{I})$ & & & 9,7 & $\begin{array}{c}10,1 \\
(0,93 \%)\end{array}$ & $\begin{array}{c}0,9 \\
(0,7 \%)\end{array}$ \\
\hline $\begin{array}{l}\text { Conductivité en } \\
\mu \mathrm{S} / \mathrm{cm} \text { à } 20^{\circ} \mathrm{C}(\mathrm{n}=\mid \mathrm{I})\end{array}$ & & & $\begin{array}{c}6450 \\
(6.8 \%)\end{array}$ & $\begin{array}{l}1736 \\
(8,7 \%)\end{array}$ & 3,7 \\
\hline $\begin{array}{l}\text { Test Algue } 72 \mathrm{~h} \\
\quad(n=17)\end{array}$ & & 100/CE50 & $\begin{array}{c}58,4 \\
(29,4 \%)\end{array}$ & $\begin{array}{c}48,6 \\
(29,6 \%)\end{array}$ & 1,2 \\
\hline $\begin{array}{l}\text { Test Microtox } 60 \mathrm{~min} . \\
\qquad(n=17)\end{array}$ & & I00/CE50 & $\begin{array}{c}5,2 \\
(21 \%)\end{array}$ & $\begin{array}{c}4,8 \\
(31,6 \%)\end{array}$ & 1,1 \\
\hline $\begin{array}{l}\text { Test Daphnie } 48 \mathrm{~h} \\
(\mathrm{n}=2)\end{array}$ & & I 100/C LC50 & $\begin{array}{l}17,4 \\
(32 \%\end{array}$ & $\begin{array}{c}26,7 \\
(8,3 \%)\end{array}$ & 0,6 \\
\hline $\begin{array}{l}\text { Test cériodaphnie } \\
7 \text { jours }(n=1)\end{array}$ & & $\begin{array}{l}1100 / C E 20 \\
1100 / C E 50\end{array}$ & $\begin{array}{l}6,7 \\
4,2\end{array}$ & $\begin{array}{l}5,7 \\
4,4\end{array}$ & $\begin{array}{l}1,17 \\
0,9\end{array}$ \\
\hline $\begin{array}{l}\text { Test plante } \\
(n=1) \\
14 \text { jours }\end{array}$ & $\begin{array}{l}\text { Germination } \\
\text { Poids frais } \\
\text { Poids sec }\end{array}$ & $\begin{array}{l}\text { 100/LOEC } \\
\text { 100/LCEC } \\
\text { 100/LOEC }\end{array}$ & $\begin{array}{c}1 \\
3,1 \\
3,13,11\end{array}$ & 1 & $\begin{array}{c}1 \\
3.1\end{array}$ \\
\hline Test d'Ames $(n=2)$ & & I00/LOEC & $N M^{* *}$ & NM & - \\
\hline Test Mutatox $(n=1)$ & & IOOILOEC & NG*** & NG & - \\
\hline
\end{tabular}

paramètres d'écotoxicité qui figurent dans le tableau 2, les séquences de toxicité pour R2 et RI0 ne sont pas statistiquement significativement différentes (test du coefficient de corrélation de rang de Spearman au seuil de 1\%). En considérant uniquement les CE ou CL50, les différents tests peuvent être classés dans l'ordre de sensibilité décrois- sante suivante: Algue > Daphnie > Microtox > Cériodaphnie. II faut enfin remarquer que le test cériodaphnie s'avère ici moins sensible que le test daphnie, ce qui est contraire aux résultats observés par Férard et Ferrari ${ }^{[1]}$ sur d'autres MIOM également fournis par Polden en 1995.

\begin{tabular}{|c|c|c|c|c|c|}
\hline \multicolumn{2}{|l|}{ Contact direct } & \multicolumn{2}{|c|}{ Mode de calcul des unités toxiques UT } & \multicolumn{2}{|c|}{ Sc2Pb solide } \\
\hline Microtox $20 \min .(n=2)$ & & \multicolumn{2}{|c|}{ 100/CE50 } & \multicolumn{2}{|c|}{$3041,5(8,6 \%)^{*}$} \\
\hline $\begin{array}{l}\text { Test plante }(n=1) \\
14 \text { jours }\end{array}$ & $\begin{array}{l}\text { Germination } \\
\text { Poids frais } \\
\text { Poids sec }\end{array}$ & $\begin{array}{l}\text { I00/LOE } \\
\text { 100/LOE } \\
\text { 100/LOE }\end{array}$ & & \multicolumn{2}{|c|}{$\begin{array}{l}55,6 \\
55,6 \\
55,6\end{array}$} \\
\hline $\begin{array}{l}\text { Contact indirect } \\
\qquad P(n=\mid 1)\end{array}$ & \multicolumn{2}{|c|}{ Mode de calcul des unités toxiques UT } & $\begin{array}{c}\mathbf{S c} 2 \mathbf{P b} 2 \mathbf{R} 2 \\
12,2\end{array}$ & $\begin{array}{c}\text { Sc2Pb2 R10 } \\
11,9^{* *}\end{array}$ & $\begin{array}{l}\mathbf{R} 2 / \mathbf{R} 10 \\
1,02\end{array}$ \\
\hline $\begin{array}{l}\text { Conductivité en } \mu \mathrm{S} / \mathrm{cm} \\
\text { à } 20^{\circ} \mathrm{C}(\mathrm{n}=4)\end{array}$ & & & $\begin{array}{l}152500 \\
(12,5 \%)\end{array}$ & $\begin{array}{l}52750 * * \\
(8,39 \%)\end{array}$ & 2,9 \\
\hline Test Algue $72 \mathrm{~h}(n=17)$ & & I00/CE50 & $\begin{array}{l}1178,5 \\
(46,9 \%)\end{array}$ & $\begin{array}{c}235,9 \\
(24,4 \%)\end{array}$ & 5 \\
\hline Test Microtox 30 min. $(n=17)$ & & I00/CE50 & $\begin{array}{l}5920,3 \\
(9,3 \%)\end{array}$ & $\begin{array}{c}1468,9 * * \\
(7,9 \%)\end{array}$ & 4 \\
\hline Test Daphnie 48 h ( $n=2)$ & & 100/CL50 & $\begin{array}{l}1080,2 \\
(43 \%)\end{array}$ & $\begin{array}{l}251.4^{* *} \\
(18,4 \%)\end{array}$ & 4,3 \\
\hline Test cériocaphnie 7 jours $(n=1)$ & & $\begin{array}{l}\text { 100/CE20 } \\
\text { I 100/CE50 }\end{array}$ & $\begin{array}{l}2881,8 \\
1883,2\end{array}$ & $\begin{array}{l}506,3 \\
355,7\end{array}$ & $\begin{array}{l}5,7 \\
5,3\end{array}$ \\
\hline $\begin{array}{l}\text { Test plante } \\
(n=1) \\
14 \text { jours }\end{array}$ & $\begin{array}{l}\text { Germination } \\
\text { Poids frais } \\
\text { Poids sec }\end{array}$ & $\begin{array}{l}\text { 100/LOEC } \\
\text { 100/LOEC } \\
\text { 100/LOEC }\end{array}$ & $\begin{array}{l}17,9 \\
17,9 \\
17,9\end{array}$ & $\begin{array}{l}5,6 \\
3,1 \\
3,1\end{array}$ & $\begin{array}{l}3,2 \\
5,8 \\
5,8\end{array}$ \\
\hline Test d'Ames $(n=2)$ & & I00/LOEC & $N M * *$ & NM & - \\
\hline Test Mutatox $(n=1)$ & & 100/LOEC & NG**** & NG & \\
\hline
\end{tabular}


- Les UT obtenues pour R2 sont légèrement plus élevées que celles obtenues pour R I0 à l'aide des tests Algues et Microtox qui ont fait l'objet de nombreuses répétitions $(n=\mid 7)$. Il faut remarquer que deux tests (daphnie et cériodaphnie) donnent des UT plus faibles pour R2 que pour RIO.

- Aucune mutagénicité ni génotoxicité n’a été détectée quel que soit le rapport L/S envisagé.

- Les tests d'écotoxicité directe sont capables, eux aussi, de révéler une toxicité relativement importante surtout en ce qui concerne le test Microtox en phase solide, caractérisé par un rapport $L / S$ de 5 (intermédiaire entre $R 2$ et $\mathrm{RIO}$ ).

\section{Cas des Sc2 Pb (tableau 3)}

- Le test Microtox est le test le plus sensible pour les $\mathrm{Sc} 2 \mathrm{~Pb}$ quelque soit le rapport L/S choisi. Le test Microtox est donc un bon indicateur de la toxicité des scories.

- Les résultats sont remarquablement concordants entre les lixiviats R2 et RIO, quels que soient les tests réalisés (le rapport varie de 3,2 à 5,8 ). En moyenne, les lixiviats $R 2$ sont 4,6 fois plus écotoxiques que les lixiviats RIO.

- En considérant uniquement les CE50 ou les CL50, les différents tests peuvent être classés dans l'ordre de sensibilité décroissante suivante : Microtox > Cériodaphnie > (Algue $\approx$ Daphnie). Le test cériodaphnie s'avère ici plus sensible que le test daphnie, contrairement à ce qui avait été observé pour les MIOM.

- Aucune mutagénicité ni génotoxicité n'a été détectée quelque soit le rapport US envisagé.

- Pour le test Microtox en phase solide, les résultats confirment la for-te toxicité observée avec le test Microtox classique. Par contre, ces résultats montrent que l'écotoxicité en phase directe est très supérieure à celle en phase indirecte pour le test plante (le facteur varie de 9,6 à 14,6).

\section{CONCLUSION}

Quel que soit le déchet étudié dans ce programme, la lixiviation avec un rapport US de 2 est généralement plus toxique que la lixiviation avec un rapport US de 10 sur la base des tests d'écotoxicité utilisés. Le test Algue apparaît comme un bon indicateur de la toxicité pour les MIOM testés, tandis que c'est le cas du test Microtox pour les Sc2Pb. De plus, les matrices testées dans ce programme apparaissent comme très différentes l'une de l'autre tant du point de vue écotoxicité indirecte ou directe. En définitive, l'ensemble des réponses obtenues indique que les $\mathrm{Sc} 2 \mathrm{~Pb}$ ont un caractère potentiellement plus dangereux que les MIOM. Enfin, les 2 déchets peuvent être considérés comme dangereux si les résultats obtenus sont confrontés aux seuils proposés par le ministère de l'Environnement $^{[12]}$.

\section{Bibliographie}

[1] Iso 11269-2. Qualité des sols Détermination des effets des polluants sur la flore du sol - Partie 2 : Effets des substances chimiques sur l'émergence et la croissance des végétaux supérieurs (1995) [2] Microbics. Microtox Solid-Phase manual, Carlsbad, Califomia (1994).

[3] Ministère de l'Environnement de l'Ontario. The fluctuation test, Watershed Management Section, Biohazard Unit (1991).

[4I Microbics. Mutatox manual, Carlsbad, California (1993)

[5] Afnor T90320. Détermination de l'inhibition de la luminescence de Photobacterium phosphoreum (1991).

[6] Iso 6341. Qualité de l'eauDétermination de l'inhibition de la mobilité de Daphnia magna Straus (Cladocera, Crustacea) - (1989).

[7I E.P.A. Short-temn methods for estimating the chronic toxicity of effluents and receiving waters to freshwater organisms. EPA 600/4-91/002 (3 $3^{\text {eme }}$ édition) (1994).
[8] Iso 8692. Qualité de l'eau - Essai d'inhibition de la croissance des algues d'eau douce avec Scenedesmus subspicatus et Selenastrum capricomutum (1989).

[9/ Biaise C., Legault R., Bermingham N., Van Coillie R. et Vasseur P. A simple microplate assay technique for aquatic toxicity assessment. Toxic. Assess. I : 26I-28I (1986).

[10] Afnor $\times 30-402$. Essai de conformité pour la lixiviation des déchets fragmentés - Étude de la lixiviation des constituants des déchets fragmentés et des boues (1996)

[II] Férard J.F. et Ferrari B. Quel test de toxicité chronique sur invertébrés fautil choisir pour l'évaluation de la dangerosité des déchets ? Déchets, Sciences \& Techniques $n^{\circ} 8$ pp. $44-47$ (1997).

[12] Ministère de l'Environnement. Critères et Méthodes d'évaluation de l'écotoxicité des déchets (1998).

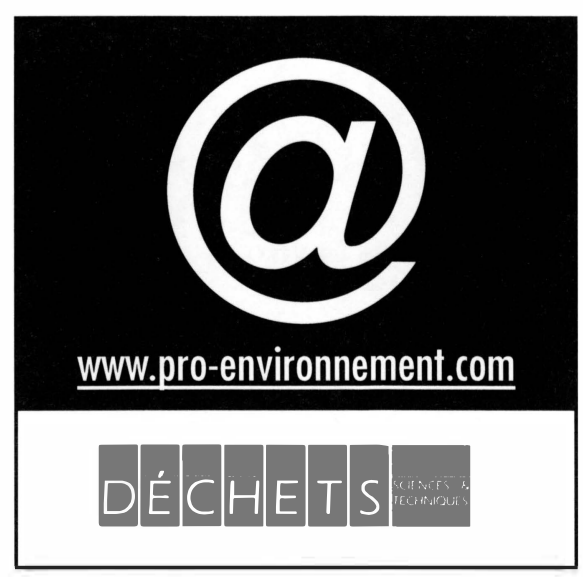

